BMJ Open Gastroenterology

\title{
Advancing the science and practice of gastroenterology and hepatology
}

Joseph K Lim

To cite: Lim JK. Advancing the science and practice of gastroenterology and hepatology. BMJ Open Gastro 2014;0:e900001. doi:10.1136/bmjgast-2014900001
Yale Liver Center, Yale University School of Medicine, New Haven, Connecticut, USA

Correspondence to Joseph K Lim; joseph.lim@yale.edu
It is my great pleasure and honour to introduce BMJ Open Gastroenterology, a new online, open access, peer-reviewed journal which will be dedicated to advancing the science and practice of gastroenterology and hepatology through the rapid publication of high-quality original research, systematic reviews, meta-analyses, endoscopic and technology reports, perspectives on all disciplines and therapeutic areas within the specialty. The creation of this journal represents a joint partnership of two great organisations with longstanding traditions of excellence in medicine, the British Society of Gastroenterology (BSG) and BMJ. Since 1840, when it was launched as the Provincial Medical and Surgical Journal, The $B M J$ has set a high standard as one of the leading authorities among medical journals, and has been distinguished for its record of ongoing innovation in medical publishing, and in fact, was the first general medical journal to enter the world wide web with the launch of bmj.com in May 1995. The BSG itself has remained a leading international specialty society within gastroenterology and hepatology, and has represented the academic and clinical home for British gastroenterologists since 1937.

At a time during which we have witnessed a significant growth in the number of specialty journals within the field of gastroenterology, why have the BSG Council and BMJ elected to pursue the launch of a new journal? Through this strategic partnership, BSG members are already twice blessed with important journals, including Frontline Gastroenterology, which under the leadership of Prof Anton Emmanuel, provides outstanding, timely, up-to-date guidance for practising clinicians with a focus on best practices and patient care, as well as Gut, which under the leadership of Prof Emad El-Omar, represents one of the most prestigious, high-impact international journals in our specialty. Due to its position as a leading if not the premier target for publication of the very best gastrointestinal research worldwide, far more papers of high quality are declined acceptance than desired due to space limitations. In fact, the manuscript acceptance rate for original research papers submitted to Gut in 2013 was a sobering 8\%. In midst of an era of flourishing research productivity and unprecedented advances in our understanding of gastrointestinal and liver diseasefrom fundamental discoveries related to the link between the gut microbiome and human disease, ${ }^{1}$ advances in biologic therapies for inflammatory bowel disorders, ${ }^{2}$ key technological breakthroughs in endoscopic therapies for obesity, ${ }^{3}$ or the rapid-fire development of all-oral interferon-free therapies for hepatitis $\mathrm{C}^{4}{ }^{5}$ - the need for additional venues for the publication of quality research has never been stronger.

It is our vision in launching BMJ Open Gastroenterology that we will share the same commitment to publishing high quality original clinical and laboratory research findings, and offer authors rapid manuscript processing and peer review, and immediate online publication. As a sister journal to Gut, we provide a mechanism through which authors of quality manuscripts which have undergone peer review but do not meet the $8 \%$ bar will be offered the option of immediate referral to BMJ Open Gastroenterology without resubmission. In this scenario, our editorial team may immediately proceed to reach a rapid editorial decision, in some cases without need for further peer review. Although we will regularly solicit editorials and reviews addressing the most timely topics in the field, we openly encourage unsolicited research manuscript submissions of all types.

The editors of BMJ Open Gastroenterology are fully committed to the principles of open access, and similar to other similar titles within the BMJ family, we aim to publish a wide range of papers not always prioritised in traditional journals, such as study protocols, phase I clinical trials, systematic reviews and meta-analyses, important negative studies, replication studies, and small or specialist studies addressing rare diseases. As an 
online-only journal, we anticipate a speedy submission and review process with continuous publication online without traditional 'issues', so as to ensure that the latest research is made available worldwide without delay. Importantly, as authors will have ownership of their papers, publications will be made available immediately online for unrestricted global access. ${ }^{6}$ Furthermore, we aim to maximise global visibility for research published in our journal through the broad traditional and social media reach of our publishing partner BMJ. We will selectively include editorials to accompany papers of high interest and broadcast paper publication through the development of press releases through BMJ. It is our full expectation that as our journal matures that we may be indexed in key literature indices including PubMed, SCOPUS, and Google Scholar, and within several years may be registered with Thomson Reuters to generate a first Impact Factor. Finally, it is my vision that this journal have strong international presence to address the needs of the global community of gastroenterology and hepatology, and hope that we will receive papers addressing the unique observations and discoveries within specific geographies which are under-represented in traditional specialty journals. We are determined to serve authors with fair and timely reviews through partnership with my three dedicated Associate Editors as well as the members of our distinguished international Editorial Board, which has representation from six continents. We enthusiastically look forward to serving you, our readers, in our launch of BMJ Open Gastroenterology.

\section{REFERENCES}

1. Arumugam M, Raes J, Pelletier E, et al. Enterotypes of the human gut microbiome. Nature 2011;473:174-80.

2. Torres J, Danese S, Colombel JF. New therapeutic avenues in ulcerative colitis: thinking out of the box. Gut 2013;62:1642-52.

3. Thompson CC, Chand B, Chen YK, et al. Endoscopic suturing for transoral outlet reduction increases weight loss after Roux-en-Y gastric bypass surgery. Gastroenterology 2013;145:129-37.

4. Afdhal N, Zeuzem S, Kwo P, et al. Ledipasvir and sofosbuvir for untreated HCV genotype 1 infection. N Engl J Med 2014; 370:1889-98.

5. Feld JJ, Kowdley KV, Coakley E, et al. Treatment of HCV with ABT-40/r-ombitasvir, and dasabuvir with ribavirin. N Engl J Med 2014;370:1594-603.

6. Wolpert AJ. For the sake of inquiry and knowledge-the inevitability of open access. N Engl J Med 2013;368:785-7. 